\title{
LA GROTTA DEL VENTO: UNA REALTA' NEL CONTESTO DEL PARCO NATURALE DELLE ALPI APUANE
}

\author{
Vittorio Verole-Bozzello*
}

\begin{abstract}
RIASSUNTO
Dopo una breve storia delle esplorazioni della grotta, viene descritta in dettaglio l'opera di turisticizzazione della grotta stessa. Questa è avvenuta cercando di salvaguardare al massimo non soltanto la sostanza dell'ambiente sotterraneo ma anche la forma con la quale il visitatore vede la grotta.

La grotta viene anche utilizzata da diversi anni, in collaborazione con la Facoltà di Medicina dell'Università di Pisa, per una interessante sperimentazione di cura dell'asma bronchiale che ha dato ottimi risultati.
\end{abstract}

\section{ABSTRACT \\ [The "Grotta del vento" (Wind Cave): \\ a feature within the Natural Park of the Apuan Alps ]}

After a short history of the explorations of the cave its development as a show cave is here reported with details. Such a development was carried out in order to preserve not only the physical integrity of the cave environment but also to keep the tourists' approach as natural as possible.

In co-operation with the Medical Faculty of the Pisa University the cave is also used since many years for an interesting and quite successful experiment for the treatment of brochial asthma.

Le Alpi Apuane, incuneate tra l'Appennino Tosco-Emiliano e la costa tirrenica, costituiscono in Italia una delle zone carsiche di maggiore interesse. L'estensione dei calcari non è omogenea, ma in più punti risulta interrotta da profonde incisioni vallive che hanno finito per toccare i sottostanti scisti impermeabili e insolubili. Lungo il piano di contatto tra i calcari e gli scisti (o poco più in alto) si aprono numerose risorgenze e sorgenti carsiche, alcune attive, altre semiattive o fossili; nelle zone più elevate abbondano gli abissi, molti dei quali veri e propri complessi sotterranei, raggiungono diverse centinaia di metri di profondità (due superano addirittura i 1000 metri) e, talvolta, diversi chilometri di sviluppo.

Il paesaggio apuano è caratterizzato da montagne aguzze e rocciose, creste affilate $e$ imponenti pareti che emergono dalla lussureggiante vegetazione delle vallate, dominando ad oriente il profilo dolce dei rilievi appenninici, ad occidente, la costa tirrenica e le isole dell'Arcipelago Toscano. Nelle giornate più limpide la vista può spaziare fino alla Corsica ed alle Alpi Marittime, mentre a settentrione, oltre le brume della Pianura Padana, è possibile scorgere le vette del Monte Bianco, del Monte Rosa, dell'Adamello, e

\footnotetext{
* c/o Grotta del Vento, I-55020 FORNOVOLASCO (Lucca) Italia
} 
In una delle zone più suggestive delle Alpi Apuane, nel Massiccio delle Panie, si apre la «Grotta del Vento», cavità carsica molto complessa che dal 1967 è attrezzata per le visite turistiche.

Conosciuta un tempo solo per il forte vento che spirava dallo stretto pertugio d'accesso, fu sfruttata per secoli dagli abitanti del vicino villaggio di Trimpello per alimentare un singolare frigorifero, costituito da una capanna dove la corrente d'aria veniva convogliata per raffreddare e conservare nei mesi estivi le vivande più deperibili.

Dopo alcuni tentativi effettuati dagli abitanti del luogo, l'esplorazione ebbe inizio nel 1932 ad opera del Gruppo Speleologico Fiorentino del C.A.I., che avanzò per soli settanta metri, arrestandosi di fronte alle acque di un sifone, superato 29 anni più tardi da speleologi di Bologna.

L'esplorazione decisiva ai fini della valorizzazione turistica fu quella compiuta dal Gruppo Speleologico Lucchese nel 1964; in quell'occasione fu percorso uno sviluppo complessivo appena superiore al chilometro, sufficiente comunque per far maturare in alcuni componenti del gruppo il desiderio di attrezzare turisticamente la grotta per offrire a tutti la possibilità di ammirare e conoscere un mondo meraviglioso altrimenti riservato alla curiosità scientifica di pochi appassionati. La valorizzazione turistica avrebbe inoltre consentito la tutela e la sorveglianza costante di un patrimonio destinato a un degrado inevitabile di cui già si potevano notare le avvisaglie nelle gallerie più facilmente accessibili; alcune concrezioni erano state infatti asportate da gente del luogo per ornare le tombe di un paese vicino.

Nell'ambiente speleologico la decisione di attrezzare turisticamente questa grotta fu accolta con un certo scetticismo: si trattava di una cavità troppo difficile, con un andamento altimetrico eccessivamente movimentato, con lunghi tratti verticali e con numerose gallerie soggette a inondazioni periodiche. Scarseggiava inoltre un requisito fondamentale secondo la corrente concezione di "grotta turistica»: la presenza di grandi ambienti pianeggianti e ricchi di concrezioni.

I fautori della turistizzazione non si scoraggiarono: «non vogliamo una grotta-vetrina», sostenevano, «di grotte turistiche orizzontali stracolme di concrezioni ne è pieno il mondo; noi, come speleologi, dobbiamo fornire al pubblico la possibilità di vivere un'esperienza diversa, basata non sulle solite immagini stereotipate e poco attinenti alla realtà, bensì sulla visione diretta e la comprensione di ogni aspetto del carsismo profondo. La valorizzazione turistica della Grotta del Vento deve assumere una funzione didattica e culturale».

E fu con questo spirito che nel 1965 tra mille difficoltà di ordine materiale e burocratico ebbero inizio i lavori. Nel $1967 \mathrm{fu}$ aperto il «primo itinerario», un percorso abbastanza lineare e «classico», costituito per la massima parte da una galleria suborizzontale abbastanza ampia e particolarmente ricca di concrezioni policrome e vive, preceduta da un centinaio di metri di condotta freatica caratterizzata da vistosi fenomeni erosivi; l'ostacolo costituito dal sifone che nel 1929 aveva fermato gli speleologi di Firenze era stato superato mediante una galleria artificiale. La visita veniva effettuata in un'ora, alla luce di maleodoranti lampade a gas portate dalle guide e dai sorveglianti. Nonostante il fatto che per raggiungere 
l'ingresso della grotta era necessaria mezz'ora di cammino lungo una ripida e assolata mulattiera, il consenso dei visitatori e della stampa fu molto lusinghiero ed incoraggiò a realizzare la seconda fase del progetto, più consona agli scopi che si prefiggevano gli speleologi valorizzatori.

Nel 1970, dal termine del «primo itinerario», il sentiero venne prolungato lungo le pareti di un baratro profondo cinquanta metri, alla base del quale, superato un tratto orizzontale ed un secondo salto di una quindicina di metri, si attraversava un salone nel quale si univano tre torrentelli, e si proseguiva lungo un'ampia galleria percorsa da un piccolo fiume sotterraneo. Il ritorno avveniva mediante un ardito sentiero che, arrampicandosi sin sul soffitto del salone, percorreva una lunga condotta freatica fossile completamente tappezzata da singolari concrezioni sabbiose e raggiungeva di nuovo il "primo itinerario». L'energia elettrica necessaria per l'impianto di illuminazione veniva fornita da due gruppi elettrogeni.

I turisti potevano scegliere tra due itinerari: il "primo" durava un'ora ed aveva un andamento abbastanza pianeggiante; il "secondo» (comprendente anche il primo) durava due ore e coi suoi strapiombi ed i suoi tratti in parete trasformava la visita in un'avventura, dando ai visitatori emozioni e sensazioni prima riservate ai soli speleologi.

Ancora una volta $\mathrm{i}$ commenti furono più che incoraggianti e negli anni successivi venne portata a termine la parte più ambiziosa dell'intero progetto: quella relativa al «terzo itinerario», che si sviluppa lungo un ramo a struttura prevalentemente verticale con incredibili sentieri sospesi sul vuoto di un pozzo a campana profondo settanta metri, percorso durante i periodi di piena da una fragorosa cascata. La visita di questo itinerario si compie in un'ora e un quarto e comprende, oltre al pozzo, gallerie finemente concrezionate, un canyon ed un'ampia sala il cui soffitto è inciso da un caratteristico solco scavato dalla regressione di una cascata.

Oggi sono disponibili tre itinerari, con durate che, variano da un minimo di un'ora (primo itinerario) ad un massimo di tre ore (visita completa)

Allo scopo di evitare gli errori compiuti altrove ed apprendere al contempo spunti ed idee utili per una valorizzazione ottimale della «Grotta del Vento", prima di iniziare i lavori fu compiuto un lungo viaggio attraverso le grotte turistiche italiane ed europee.

Per quanto concerne $i$ sentieri, nella «Grotta del Vento» si è accuratamente evitato di spianare o di inghiaiare il pavimento delle gallerie, soluzione altrove adottata allo scopo di occultare o rendere più «naturali» $i$ camminamenti. I sentieri sono stati realizzati in cemento, avendo cura di evitare nella maniera più assoluta il danneggiamento delle concrezioni e dei particolari morfologici più significativi; anche il contatto con la roccia delle pareti e del pavimento è stato evitato il più possibile, ricorrendo spesso a passerelle sospese. La larghezza media dei tratti di scorrimento è inferiore al sessanta centimetri; ciò oltre a limitare l'impatto ambientale, consente ai visitatori di appoggiarsi a entrambe le ringhiere, a tutto vantaggio della sicurezza. Ovviamente $\mathrm{i}$ tratti di scorrimento sono intervallati a zone più ampie, destinate all'incrocio e alla sosta dei gruppi.

Nella realizzazione dei sentieri più recenti si sono preferite le linee curve alle linee spezzate e le ringhiere, eseguite in acciaio zincato, seguono 
perfettamente l'andamento dei bordi in modo che sia praticamente impossibile mettere un piede in fallo. L'acciaio zincato è stato preferito ad altri materiali per il suo colore neutro, per la sua curvabilità e per la sua relativa inossidabilità. L'acciaio verniciato non regge alla corrosione, l'acciaio inossidabile risulta troppo brillante, mentre le materie plastiche, benchè più piacevoli al tatto, risultano scarsamente modellabili consentendo la realizzazione soltanto di segmenti di retta. Per le gradinate sono stati preventivamente calcolati i raggi di curvatura, egualizzando le alzate e le pedate dei gradini in modo da offrire il massimo comfort anche alle persone anziane.

Il sentiero ottimale dovrebbe consentire ai visitatori di procedere senza essere costretti a guardare in continuazione dove mettere $i$ piedi. In tal modo tutta l'attenzione può essere rivolta all'osservazione dell'ambiente ed all'apprendimento di quanto viene esposto dalle guide.

L'impianto elettrico evita accuratamente sia l'«effetto presepe» (troppe piccole luci che punteggiano l'ambiente), sia l'«effetto vetrina» (illuminazione sfarzosa senza zone d'ombra). I punti-luce, sempre costituiti da lampade ad incandescenza, lasciano in ombra ampie zone povere di particolari interessanti allo scopo di rispettare quel senso di mistero che l'ambiente sotterraneo deve suscitare nel visitatore.

Le lampade ad incandescenza sono state preferite a quelle «a scarica di gas" (vapori di mercurio, neon, vapori di sodio ad alta o bassa pressione, joduri metallici) sia per la loro migliore o comunque più gradevole resa cromatica, sia perchè il raggiungimento della piena potenza luminosa è immediato. Per evitare la formazione di muschi, felci ed alghe verdi, oltre che per ovvi motivi di risparmio energetico, l'impianto è stato frazionato in vari tratti in modo da limitare il più possibile il tempo di accensione di ogni singolo tratto.

Particolare cura viene posta nell'addestramento delle guide, che prima di cominciare a fornire spiegazioni al pubblico debbono seguire un corso di preparazione che le pone in grado di rispondere con precisione e competenza alla maggior parte dei quesiti che possono essere posti dai visitatori. La spiegazione si basa in gran parte sulle domande dei visitatori: ciò vivacizza l'esposizione degli argomenti, che altrimenti rischiano di venire trattati in maniera sempre più piatta, adottando sempre lo stesso ordine e lo stesso frasario. Una guida non può limitare la propria conoscenza ad un minimo di nozioni indispensabili per la spiegazione; solo sapendo molto di più di ciò che si dice, è possibile variare l'esposizione degli argomenti adattandola alle esigenze ed al tipo di interesse dei visitatori.

Un'altra qualità essenziale per una buona guida è la capacità di comunicare agli altri il proprio entusiasmo per il mondo sotterraneo; per raggiungere questo scopo non basta provare interesse o amore per le grotte, ma è necessario immedesimarsi nello stato d'animo dei visitatori cercando ogni volta di riscoprire la grotta attraverso i loro occhi.

Negli ultimi anni sono stati organizzati più volte viaggi di istruzione $\mathrm{e}$ aggiornamento per le guide, che in tal modo hanno potuto visitare numerose altre grotte turistiche. Ciò consente di inserire nella spiegazione numerosi riferimenti alle altre cavità, spronando la gente ad allargare la propria 
conoscenza sul patrimonio speleoturistico esistente. L'importante è far capire che non esiste una grotta migliore di tutte le altre, ma che ogni grotta, anche la più piccola, può avere qualcosa che le altre non hanno. In tal modo i visitatori saranno spinti ad osservare le grotte con occhi diversi, tornando più volte nelle stesse grotte per confrontarle attentamente con ciò che hanno visto nelle altre. Se, al contrario, si cercherà di presentare una grotta come «la migliore del mondo», i visitatori, appagati dalla convinzione di aver visto «il massimo», esauriranno con la prima esperienza il loro interesse per il mondo sotterraneo.

La presenza dei sentieri e dell'energia elettrica facilita sensibilmente l'esplorazione, tuttora in corso su varie direttrici. Alcuni sifoni sono stati superati prosciugandoli con elettropompe, l'arrampicata sulle pareti verticali viene facilitata dall'impiego dei trapani elettrici, mentre gli «ancoraggi chimici» rendono sicura la progressione anche su rocce molto friabili.

In campo scientifico è stata particolarmente curata la ricerca nel campo geo-morfologico, idrologico, meteorologico e microclimatico. Sotto l'egida della Facoltà di Medicina dell'Università di Pisa, è in corso da diversi anni una interessante sperimentazione speleoterapica che ha consentito di rilevare su decine di pazienti risultati talvolta sbalorditivi nella cura dell'asma bronchiale.

Due diramazione della grotta sono state attrezzate specificamente per questo scopo ed ospitano per periodi di ventun giorni gruppi di pazienti che sotto la costante sorveglianza medica permangono nell'ambiente sotterraneo per tempi variabili dalle tre alle otto ore al giorno. La sperimentazione sui pazienti è stata preceduta ed affiancata da un'accurata ricerca microclimatica effettuata mediante l'analisi periodica di campioni di aria e di acqua prelevati dalla grotta e comparati con campioni prelevati all'esterno. Nel contempo, sono stati compiuti anche esperimenti sui ratti bianchi.

In un'altra diramazione è in fase di allestimento una stazione di rilevamento sismico, permanentemente collegata via telefono al calcolatore dell'Osservatorio sismografico di Prato. Data l'elevata sismicità della Garfagnana, l'importanza di questa stazione assume un'importanza di tutto rilievo.

Tutto ciò conferma quanto sia errato il giudizio di molti speleologi e di molti ambientalisti $\mathrm{i}$ quali sostengono che ogni grotta turistica è una grotta persa, quando invece possiamo affermare che, al contrario, ogni grotta turistica può essere una grotta acquisita per la ricerca scientifica e per la conoscenza più approfondita del mondo sotterraneo.

L'ingresso della grotta è situato al centro di un'area di eccezionale interesse paesaggistico e morfologico. La zona di assorbimento che alimenta il sistema, situata attorno ai 1400 metri di quota è caratterizzata da vistosi fenomeni carsici di superficie, con estesi campi solcati, doline, voragini (tra le quali un abisso a salto unico di oltre 300 metri), nevai sotterranei e singolari forme di corrosione superficiale quali le scannellature a lisca di pesce, i solchi a doccia e le kamenitze. Sempre nella stessa zona è possibile ammirare un gigantesco arco naturale che collega le due vette gemelle di un monte. Per chi è disposto a percorrere qualche ora a piedi esiste quindi la possibilità di integrare la visita dell'ambiente sotterraneo con un'affascinante escursione 
tragli aspetti più singolari del carsismo di superficie.

Presso l'ingresso è stato eretto un ampio edificio che ospita la biglietteria, i servizi igienici, una grande esposizione di minerali e di fossili ed un accogliente punto di ristoro.

Oggi la "Grotta del Vento» è raggiungibile mediante una strada asfaltata realizzata in gran parte a spese dell'Amministrazione della grotta stessa. Le aree di parcheggio consentono la sosta ad un centinaio di auto. Sono in corso d'opera $\mathrm{i}$ lavori di ampliamento di alcune curve che attualmente non consentono il passaggio dei pullman aventi una lunghezza superiore agli 11 metri. Si tratta comunque di interventi modesti e tardivi, operati da una pubblica amministrazione che non comprende o non vuol comprendere appieno l'enorme importanza che la grotta, massimo punto di attrazione della Garfagnana e delle Alpi Apuane, potrebbe assumere nello sviluppo economico di tutta la zona.

A complicare ulteriormente le cose, la recente istituzione del Parco Naturale delle Alpi Apuane, impone enormi limiti e pastoie burocratiche alla realizzazione di qualsiasi intervento si possa rendere necessario per migliorare la fruibilità del territorio e le condizioni di vita della popolazione locale. 